Symmetries, Differential Equations and Applications (SDEA-II)

International Journal of Modern Physics: Conference Series

Vol. 38 (2015) 1560080 (12 pages)

(C) The Author

DOI: $10.1142 / \mathrm{S} 2010194515600800$

\title{
A finite difference approach to find exact solution of differential equations
}

\author{
Sergei Zuev \\ Department of the Computer Software and Automated Systems \\ Belgorod State Technological University named after V.G. Shoukhov \\ 46 Kostyukova Str. Belgorod, 308012, Russia \\ sergey.zuev@bk.ru
}

Published 30 April 2015

\begin{abstract}
This paper contains the background and samples of an approach to construct exact solutions of a wide range of differential equations (DEs). This approach is based on the finite difference equation which corresponds to the given DE. There are three cases considered: linear partial differential equation (PDE) with constant coefficients and at least one non-zero root of characteristic equation, linear PDE with constant coefficients and completely zero roots of the characteristic equation, and a case of nonlinear autonomous dynamical system of second order. Each of these cases is illustrated by an example.
\end{abstract}

Keywords: Finite difference equation; exact solutions; linear PDE; nonlinear autonomous dynamical systems.

\section{Introduction}

There exist many physical systems that have a differential equation description that looks quite simple. However it is difficult to find the exact expression for a motion and even to define the phase trajectories of such simple systems. For example, the general consideration of a one-dimensional heat equation and the corresponding Cauchy problem was carried out by Canon (1984). ${ }^{1}$ Instead of finding the exact solution of differential equations which could be very challenging at times, it is now common to investigate properties of the physical systems using other schemes. For example one such approach is to examine the solution of DEs by numerical techniques. But in some cases it is not enough to only know the solution behavior and its numerical solution; it is critical to find the exact solution. For instance, in some problems of hydrodynamics ${ }^{2}$ where the basic PDE is non-linear, the system requires that the condition of continuity (linear PDE) must be obeyed. In a dynamical system with nonlinear ODE, there are also many open problems and challenges. In

This is an Open Access article published by World Scientific Publishing Company. It is distributed under the terms of the Creative Commons Attribution 3.0 (CC-BY) License. Further distribution of this work is permitted, provided the original work is properly cited. 
particular, the well-known van der Pol oscillator ${ }^{3-}, 4$ which represents the simplest model with dynamic chaos, has no exact solution yet.

As a proposition, in the nonlinear case, the linear nature in differentiation itself could be a source of difficulties. As for the linear PDE case, the reason for difficulties could be a broken scale invariance of the system. Both of these issues can be overcome by means of finite difference equations (FDE) or, according to another definition, lattice equations.

Remark 1.1. For any function $u\left(x_{1}, \ldots, x_{n}\right)$ on $n$ real variables $x_{l}, l=1, \ldots, n$, in some neighborhood of the point $p_{0}=\left(x_{01}, \ldots, x_{0 n}\right)$ which contains point $\left(x_{01}, \ldots, x_{0 l}+\tau_{l}, \ldots, x_{0 n}\right)$, the finite difference derivation in $p_{0}$ with respect to $x_{l}$ is defined as

$$
D_{x_{l}} u\left(p_{0}\right)=\frac{u\left(x_{01}, \ldots, x_{0 l}+\tau_{l}, \ldots, x_{0 n}\right)-u\left(x_{01}, \ldots, x_{0 n}\right)}{\tau_{l}} .
$$

Remark 1.2. Any functional equation which contains finite difference derivation is called finite difference equation.

The first order FDE has the following general form

$$
F\left(D_{x_{1}} u, \ldots, D_{x_{n}} u, u, x_{1}, \ldots, x_{n}\right)=0 .
$$

This equation determines the value $u\left(x_{1}+\tau_{1}, \ldots, x_{n}+\tau_{n}\right)$ of the unknown function in the neighborhood of the point $p_{0}$ when the initial and boundary conditions are specified.

The $\tau_{l}$ parameters are minimal values of the finite differences. Eq. (2) transforms to PDE when $\tau_{l} \forall l$, tend to zero and $\lim _{\tau_{l} \rightarrow 0} D_{l} u$, exist $\forall l$. If the boundary conditions are given, then the function $u\left(x_{1}, \ldots, x_{n}\right)$ inside the boundary is determined by that PDE. Therefore, when the unknown function is differentiable (this is necessary for PDE formulation), it is possible to find a solution of PDE

$$
v \equiv \lim _{\tau_{1}, \ldots, \tau_{n}=0} u
$$

from the solution $u$ of FDE if all the relevant limits exist.

For linear PDE the following theorem holds.

Theorem 1.1. If Eq. (2) is linear, i.e.

$$
\sum_{i=1}^{n} P_{i}\left(x_{1}, \ldots, x_{n}\right) D_{x_{i}} u+Q\left(x_{1}, \ldots, x_{n}\right) u+R\left(x_{1}, \ldots, x_{n}\right)=0,
$$

then it's solutions are in one-to-one correspondence with solutions $v\left(x_{1}, \ldots, x_{n}\right)$ of the linear PDE

$$
\sum_{i=1}^{n} P_{i}\left(x_{1}, \ldots, x_{n}\right) \frac{\partial v}{\partial x_{i}}+Q\left(x_{1}, \ldots, x_{n}\right) v+R\left(x_{1}, \ldots, x_{n}\right)=0,
$$

and $\lim _{\tau_{1}, \ldots, \tau_{n}=0} u=v$. 
Proof. When $\tau_{1}, \ldots, \tau_{n}$ go to zero, Eq. (4) becomes Eq. (5) and $v=\lim _{\tau_{1}, \ldots, \tau_{n}=0} u$ obeys Eq. (5). Therefore it is evident that for any $u$ we can find $v$ as the Theorem requires.

Now we prove the converse statement. Let us do it for $n=2$ (for arbitrary $n$ it can be made by analogy). Eqs. (4) and (5) will take the forms:

$$
P_{1} D_{1} u+P_{2} D_{2} u+Q u+R=0
$$

and

$$
P_{1} \partial_{1} v+P_{2} \partial_{2} v+Q v+R=0 .
$$

Let $v$ is given and obeys Eq. (7). Let us find $u$ which has the $\operatorname{limit}_{\lim _{\tau_{1}, \tau_{2} \rightarrow 0} u=v}$ and in the same time obeys (6).

We can represent $u$ as

$$
u=\sum_{i, j=0}^{\infty} \tau_{1}^{i} \tau_{2}^{j} u_{i j}\left(x_{1}, x_{2}\right), \quad u_{00} \equiv v
$$

From here

$$
\partial_{1}^{k} u=\sum_{i, j=0}^{\infty} \tau_{1}^{i} \tau_{2}^{j} \partial_{1}^{k} u_{i j}\left(x_{1}, x_{2}\right), \quad \partial_{2}^{k} u=\sum_{i, j=0}^{\infty} \tau_{1}^{i} \tau_{2}^{j} \partial_{2}^{k} u_{i j}\left(x_{1}, x_{2}\right)
$$

for any $k \in \mathbf{N}$. Since $\tau_{1}$ and $\tau_{2}$ are small, it is possible to expand function $u$ to the Taylor range near $\left(x_{1}, x_{2}\right)$ :

$$
\begin{aligned}
& u\left(x_{1}+\tau_{1}, x_{2}\right)=u\left(x_{1}, x_{2}\right)+\partial_{1} u \frac{\tau_{1}}{1 !}+\partial_{1}^{2} u \frac{\tau_{1}^{2}}{2 !}+\ldots \\
& u\left(x_{1}, x_{2}+\tau_{2}\right)=u\left(x_{1}, x_{2}\right)+\partial_{2} u \frac{\tau_{2}}{1 !}+\partial_{2}^{2} u \frac{\tau_{2}^{2}}{2 !}+\ldots
\end{aligned}
$$

Therefore

$$
D_{1} u=\partial_{1} u+\partial_{1}^{2} u \frac{\tau_{1}}{2 !}+\ldots, \quad D_{2} u=\partial_{2} u+\partial_{2}^{2} u \frac{\tau_{2}}{2 !}+\ldots
$$

Substituting it into Eq. (6) and writing down terms with factors $\tau_{1}^{i} \tau_{2}^{j}$, we get

$$
\begin{array}{ll}
i=0, j=0: & P_{1} \partial_{1} v+P_{2} \partial_{2} v+Q v+R=0, \\
i=1, j=0: & P_{1}\left(\partial_{1} u_{10}+\frac{1}{2} \partial_{1}^{2} v\right)+P_{2}\left(\partial_{2} u_{10}+\frac{1}{2} \partial_{2}^{2} v\right)+Q u_{10}=0,
\end{array}
$$

and so on. The first equation of this series is exactly Eq. (6). The others equations determine all functions $u_{i j}$. So we have found all components in the expansion given by Eq. (8). The function $u$ is completely determined.

Similar propositions were presented by Gelfond. ${ }^{5}$ So, for the linear case, the functions $u$ and $v$, connected by Eq. (3), always exist. That is why we have the following approach to find exact solution of linear PDE: 
- Write down FDE from the given PDE by just changing derivatives to finite difference derivatives;

- Solve FDE and find the form of $u\left(x_{1}+\tau_{1}, \ldots, x_{n}+\tau_{n}\right)$;

- Let all differences $\tau_{l}$ tend to zero and find the solutions of the linear PDE by calculating its limits.

The above results for FDEs of scalar functions can easily be generalized to vector-functions. In which case, we need to examine a system of equations instead of a single equation.

For FDE, it is normally assumed that the subject is numerical. In reality it is not the case. For example in the case of linear FDE, once it is possible to find exact FDE solution then we can get the exact solution of the linear PDE which is evident from above. This is the core of the proposed approach in this paper.

\section{Linear PDE-I: Heat equation}

Let us consider the case of a homogeneous rod without any heat sources, modeled by the heat equation

$$
\frac{\partial u}{\partial t}-\alpha \frac{\partial^{2} u}{\partial x^{2}}=0
$$

which we solve by using the above mentioned approach. As a first step, write down the FDE assuming that all the derivatives in Eq. (9) become finite. In general, the finite difference values are different for $x$ and $t$. Let us denote the finite differences of $x$ and $t$ with $\xi$ and $\tau$, respectively. Using these notations,

$$
y=\frac{\tau}{\xi} x
$$

we find the FDE which corresponds to the same differential equation (9)

$$
D_{t} u-\beta D_{y}^{2} u=0,
$$

where $\beta=\alpha \tau^{2} / \xi^{2}$. The differences for both independent variables is equal to $\tau$. This equation is linear and the linear combination of its solutions is the solution. Now we consider the following situation, the ranges of the values of unknown function for both independent variables coincide with each other while the other variable is fixed. This allows us to find the solution of (10) in the following form

$$
\begin{aligned}
u(t, y)= & C_{1}\left(\tau \lambda_{1}+1\right)^{\frac{t}{\tau}}\left(\tau \lambda_{1}+1\right)^{\frac{y}{\tau}}+C_{2}\left(\tau \lambda_{1}+1\right)^{\frac{t}{\tau}}\left(\tau \lambda_{2}+1\right)^{\frac{y}{\tau}} \\
& +C_{3}\left(\tau \lambda_{2}+1\right)^{\frac{t}{\tau}}\left(\tau \lambda_{1}+1\right)^{\frac{y}{\tau}}+C_{4}\left(\tau \lambda_{2}+1\right)^{\frac{t}{\tau}}\left(\tau \lambda_{2}+1\right)^{\frac{y}{\tau}},
\end{aligned}
$$

where $\lambda_{1}$ and $\lambda_{2}$ are solutions of the characteristic equation

$$
\lambda-\beta \lambda^{2}=0,
$$

which has the following roots

$$
\lambda_{1}=0, \lambda_{2}=\frac{1}{\beta} .
$$


The solution will take the form

$$
u(t, y)=C_{1}+C_{2}\left(\frac{\tau}{\beta}+1\right)^{\frac{y}{\tau}}+C_{3}\left(\frac{\tau}{\beta}+1\right)^{\frac{t}{\tau}}+C_{4}\left(\frac{\tau}{\beta}+1\right)^{\frac{t}{\tau}}\left(\frac{\tau}{\beta}+1\right)^{\frac{y}{\tau}}
$$

Substituting this into Eq. (10), we get

$$
C_{2}=C_{3}=0
$$

while $C_{1}, C_{4}$ are arbitrary constants. Finally

$$
u(t, y)=C_{1}+C_{4}\left(\frac{\tau}{\beta}+1\right)^{\frac{t}{\tau}}\left(\frac{\tau}{\beta}+1\right)^{\frac{y}{\tau}} .
$$

Now, recall that $\beta=\alpha \frac{\tau^{2}}{\xi^{2}}$ and $y=\frac{\tau}{\xi} x$, therefore

$$
u(t, x)=C_{1}+C_{4}\left(\frac{\xi^{2}}{\alpha \tau}+1\right)^{\frac{t}{\tau}+\frac{x}{\xi}} .
$$

This is the exact solution of finite difference heat equation for homogeneous rod without heat sources. However, $\tau$ and $\xi$ are still undetermined. To get the solution of corresponding PDE it is necessary to put $\tau, \xi \rightarrow 0$. Let us denote

$$
\lim _{\tau, \xi \rightarrow 0} \frac{\xi}{\tau}=k .
$$

After taking the limit we arrive at the solution

$$
u(t, x)=C_{1}+C_{4} e^{\frac{k^{2} t}{\alpha}} e^{\frac{k x}{\alpha}} .
$$

The known general solution of the heat equation is determined for $x \in(0 ; L)$, $\tilde{u}(0, x)=\phi(x)$ and $\tilde{u}(t, 0)=\tilde{u}(t, L)=0$ and presented in Cannon ${ }^{1}$ as

$$
\tilde{u}(t, x)=\sum_{n=1}^{\infty}\left(\frac{2}{L} \int_{0}^{L} \phi(\xi) \sin \left(\frac{\pi n}{l} \xi\right) d \xi\right) \sin \left(\frac{\pi n}{L} x\right) e^{-\alpha\left(\frac{\pi n}{L}\right)^{2} t} .
$$

If we put $k_{n}=i \alpha \frac{\pi n}{L}$ in Eq. (13), then this solution will be the imaginary part of the sum of the solutions of Eq. (13) form, i.e.

$$
\tilde{u}(t, x)=\sum_{n=1}^{\infty} A_{n} e^{\frac{k_{n}^{2} t}{\alpha}} e^{\frac{k_{n} x}{\alpha}}
$$

where

$$
A_{n}=\frac{2}{L} \int_{0}^{L} \phi(\xi) \sin \left(\frac{\pi n}{L} \xi\right) d \xi .
$$

This above example illustrates the approach in the case when the characteristic equation has at least one non-zero root. This result was published in Russian by Dorokhova. ${ }^{6}$ 


\section{Linear PDE-II: Homogeneous continuity equation}

\subsection{The problem description}

A well-known PDE, which represents the case of zero eigenvalue is the homogeneous continuity equation

$$
\frac{\partial v_{x}}{\partial x}+\frac{\partial v_{y}}{\partial y}+\frac{\partial v_{z}}{\partial z}=0
$$

This equation determines the so called solenoidal vector fields

$$
\mathbf{v}(x, y, z)=\left(v_{x}(x, y, z), v_{y}(x, y, z), v_{z}(x, y, z)\right)
$$

in the set $D \subseteq \mathbf{R}^{3}$, where the functions $v_{x}, v_{y} v_{z}$ are continuously differentiable at least once.

In this section the general solution of Eq. (14) is constructed for the vector fields which are determined over $\mathbf{R}^{3}$ and obey the physical assumptions expressed in Carlson $^{2}$ (a further application of the solution in Euler and Navier-Stokes equations). These assumptions are:

(1) vector field $\mathbf{v}$ is determined, continuous and differentiable on the whole $\mathbf{R}^{3}$;

(2) vector field $\mathbf{v}$ obeys the condition of limited energy: $\int_{\mathbf{R}^{3}}|v(x, y, z, t)|^{2} d x d y d z<$ $C$ for any given $t$.

We follow the same procedure to obtain the solution of above equation with respect to the linearity of Eq. (14).

\subsection{FDE for homogeneous continuity equation}

The FDE corresponding to (14) is

$$
D_{x} u_{x}+D_{y} u_{y}+D_{z} u_{z}=0,
$$

where $D_{x}$ is finite difference derivation for $x$ (the same for $y$ and $z$ ). The name of the vector field components is changed to $u$ in order to separate it from the target field $\mathbf{v}$ which is the solution of Eq. (14).

In the introduction we mentioned that in the case of linear PDE the reason of difficulties may be connected with broken scale invariance of the system. The exponential change in the dependent variable allows us to avoid this breaking of scale invariance using new unknown functions. That is why the components of the vector field $\mathbf{u}$ are re-defined in terms of the exponential counterparts

$$
u_{l}=e^{\phi_{l}}, \quad l=x, y, z .
$$

The functions $\phi_{l}$, are now $\mathbf{C}$-valued because $u_{l}$ might be negative. Moreover, $\phi_{l}$ are also multi-valued, i.e., if their values change by $2 \pi k i, k \in \mathbf{Z}, i^{2}=-1$, then we get the same values of $u_{l}$. The finite difference derivations $D_{l} u_{l}$ in the new variables take the form

$$
D_{l} u_{l}=e^{\phi_{l}} \frac{e^{D_{l} \phi_{l} \tau_{l}}-1}{\tau_{l}},
$$


and Eq. (15) becomes

$$
\tau_{y} \tau_{z}\left(e^{\phi_{x}+D_{x} \phi_{x} \tau_{x}}-e^{\phi_{x}}\right)+\tau_{x} \tau_{z}\left(e^{\phi_{y}+D_{y} \phi_{y} \tau_{y}}-e^{\phi_{y}}\right)+\tau_{x} \tau_{y}\left(e^{\phi_{z}+D_{z} \phi_{z} \tau_{z}}-e^{\phi_{z}}\right)=0 .
$$

The main idea to find solutions to above equation is to explore exponent indices

$$
\phi_{x} ; \phi_{y} ; \phi_{z} ; \phi_{x}+D_{x} \phi_{x} \tau_{x} ; \phi_{y}+D_{y} \phi_{y} \tau_{y} ; \phi_{z}+D_{z} \phi_{z} \tau_{z}
$$

From a simple observation we note that each of these indices can have one of the two possible forms, constant or variable, one can derive that there are only 7 possible sets $\left\{\phi_{l}\right\}$ :

(1) $\phi_{x}=c_{x}, \phi_{y}=c_{y}, \phi_{z}=\sigma_{z}(x, y, t)$

(2) $\phi_{x}=c_{x}, \phi_{y}=\sigma_{y}(x, z, t), \phi_{z}=c_{z}$

(3) $\phi_{x}=\sigma_{x}(y, z, t), \phi_{y}=c_{y}, \phi_{z}=c_{z}$

(4) $\phi_{x}=c_{x}, \phi_{y}=c_{y} \frac{y}{\tau_{y}}+c_{z} \frac{z}{\tau_{z}}+\sigma_{y z}(x, t), \phi_{z}=\phi_{y}+\ln \frac{\tau_{z}\left(1-e^{c_{y}}\right)}{\tau_{y}\left(e^{\left.c_{z}-1\right)}\right.}$

(5) $\phi_{x}=c_{x} \frac{x}{\tau_{x}}+c_{z} \frac{z}{\tau_{z}}+\sigma_{x z}(y, t), \phi_{y}=c_{y}, \phi_{z}=\phi_{x}+\ln \frac{\tau_{z}\left(1-e^{c_{x}}\right)}{\tau_{x}\left(e^{c_{z}}-1\right)}$

(6) $\phi_{x}=\phi_{y}+\ln \frac{\tau_{x}\left(1-e^{c y}\right)}{\tau_{y}\left(e^{c_{x}}-1\right)}, \phi_{y}=c_{y} \frac{y}{\tau_{y}}+c_{x} \frac{x}{\tau_{x}}+\sigma_{y x}(z, t), \phi_{z}=c_{z}$

(7) $\phi_{x}=c_{x} \frac{x}{\tau_{x}}+c_{y} \frac{y}{\tau_{y}}+c_{z} \frac{z}{\tau_{z}}+\psi_{x}(t)$,

$$
\phi_{y}=c_{x} \frac{x}{\tau_{x}}+c_{y} \frac{y}{\tau_{y}}+c_{z} \frac{z}{\tau_{z}}+\psi_{y}(t), \phi_{z}=c_{x} \frac{x}{\tau_{x}}+c_{y} \frac{y}{\tau_{y}}+c_{z} \frac{z}{\tau_{z}}+\psi_{z}(t),
$$

and the following condition applies

$$
\tau_{z} \tau_{y} e^{\psi_{x}(t)}\left(e^{c_{x}}-1\right)+\tau_{x} \tau_{z} e^{\psi_{y}(t)}\left(e^{c_{y}}-1\right)+\tau_{x} \tau_{y} e^{\psi_{z}(t)}\left(e^{c_{z}}-1\right) .
$$

The sets (1)-(3) lead to the solution of Eq. (15) in the form

$$
\mathbf{u}^{(1)}=\left(\begin{array}{c}
f_{x}(y, z, t) \\
f_{y}(x, z, t) \\
f_{z}(x, y, t)
\end{array}\right)
$$

which appear in any other solutions as a sum.

The sets (4)-(6) give the solutions

$$
\mathbf{u}^{(2)}=\left(\begin{array}{l}
f_{x z}(y, t) e^{\frac{c_{x 2} x}{\tau_{x}}} e^{\frac{c_{z 2} z}{\tau_{z}}}+\frac{\tau_{x}\left(1-e^{c_{y 3}}\right)}{\tau_{y}\left(e^{\left.c_{x 3}-1\right)}\right.} f_{y x}(z, t) e^{\frac{c_{y 3} y}{\tau_{y}}} e^{\frac{c_{x 3} x}{\tau_{x}}} \\
f_{y z}(x, t) e^{\frac{c_{y 1} y}{\tau_{y}}} e^{\frac{c_{z 1} z}{\tau_{z}}}+f_{y x}(z, t) e^{\frac{c_{y 3} y}{\tau_{y}}} e^{\frac{c_{x 3} x}{\tau_{x}}} \\
\frac{\tau_{z}\left(1-e^{c_{y 1}}\right)}{\tau_{y}\left(e^{\left.c_{z 1}-1\right)}\right.} f_{y z}(x, t) e^{\frac{c_{y 1} y}{\tau_{y}}} e^{\frac{c_{z 1} z}{\tau_{z}}}+\frac{\tau_{z}\left(1-e^{c_{x 2}}\right)}{\tau_{x}\left(e^{\left.c_{z 2}-1\right)}\right.} f_{x z}(y, t) e^{\frac{c_{x 2} x}{\tau_{x}}} e^{\frac{c_{z 2} z}{\tau_{z}}}
\end{array}\right),
$$

where $c_{i j}$ are constants.

As for Eq. (7), the solution is

$$
\mathbf{u}^{(3)}=e^{\frac{c_{x} x}{\tau_{x}}} e^{\frac{c_{y} y}{\tau_{y}}} e^{\frac{c_{z} z}{\tau_{z}}}\left(\begin{array}{l}
g_{x}(t) \\
g_{y}(t) \\
g_{z}(t)
\end{array}\right)
$$

The general solution of Eq. (15) is the sum $\mathbf{u}^{(1)}+\mathbf{u}^{(2)}+\mathbf{u}^{(3)}$ and with the condition

$$
g_{x}(t) \frac{e^{c_{x}}-1}{\tau_{x}}+g_{y}(t) \frac{e^{c_{y}}-1}{\tau_{y}}+g_{z}(t) \frac{e^{c_{z}}-1}{\tau_{z}}=0
$$

for every summand (range indices are omitted for clarity). 


\subsection{The PDE solution}

Let us denote

$$
\begin{aligned}
& q_{x}=\frac{e^{c_{x}}-1}{\tau_{x}}, q_{y}=\frac{e^{c_{y}}-1}{\tau_{y}}, q_{z}=\frac{e^{c_{z}}-1}{\tau_{z}} \\
& q_{x j}=\frac{e^{c_{x j}}-1}{\tau_{x}}, q_{y j}=\frac{e^{c_{y j}}-1}{\tau_{y}}, q_{z j}=\frac{e^{c_{z j}}-1}{\tau_{z}}, j=1,2,3 .
\end{aligned}
$$

Now let $\tau_{x}, \tau_{y}, \tau_{z} \rightarrow 0$ and every summand of the solution of (14) will be the limit of the sum

$$
\mathbf{u}=\lim _{\tau_{x}, \tau_{y}, \tau_{z} \rightarrow 0} \mathbf{u}^{(1)}+\mathbf{u}^{(2)}+\mathbf{u}^{(3)}
$$

More precisely

$$
\begin{aligned}
u_{x}= & g_{x}(t) e^{q_{x} x+q_{y} y+q_{z} z}+f_{x z}(y, t) e^{q_{x 2} x+q_{z 2} z} \\
& -\frac{q_{y 3}}{q_{x 3}} f_{y x}(z, t) e^{q_{y 3} y+q_{x 3} x}+f_{x}(y, z, t) \\
u_{y}= & g_{y}(t) e^{q_{x} x+q_{y} y+q_{z} z}+f_{y z}(x, t) e^{q_{y 1} y+q_{z 1} z} \\
& +f_{y x}(z, t) e^{q_{y 3} y+q_{x 3} x}+f_{y}(x, z, t) \\
u_{z}= & g_{z}(t) e^{q_{x} x+q_{y} y+q_{z} z}-\frac{q_{y 1}}{q_{z 1}} f_{y z}(x, t) e^{q_{y 1} y+q_{z 1} z} \\
& -\frac{q_{x 2}}{q_{z 2}} f_{x z}(y, t) e^{q_{x 2} x+q_{z 2} z}+f_{z}(x, y, t) .
\end{aligned}
$$

The Eq. (20) takes the form

$$
g_{x}(t) q_{x}+g_{y}(t) q_{y}+g_{z}(t) q_{z}=0
$$

for every summand.

After integrating (21) over $\mathbf{R}^{3}$, the final derivation comes out to be

$$
\mathbf{v}=\left(\begin{array}{c}
G_{x}+F_{1}-H_{2}+f_{x}(y, z, t) \\
G_{y}+F_{3}+F_{2}+f_{y}(x, z, t) \\
G_{z}-H_{3}-H_{1}+f_{z}(x, y, t)
\end{array}\right),
$$

where functions $G_{x}, G_{y}, G_{z}, F_{1}, F_{2}, F_{3}, H_{1}, H_{2}, H_{3}$ are determined as follows:

$$
\begin{aligned}
G_{x}= & \int_{\mathbf{R}^{3}} g_{x}(\alpha, \beta, \gamma, t) e^{2 \pi i(\alpha x+\beta y+\gamma z)} d \alpha d \beta d \gamma, \\
G_{y}= & \int_{\mathbf{R}^{3}} g_{y}(\alpha, \beta, \gamma, t) e^{2 \pi i(\alpha x+\beta y+\gamma z)} d \alpha d \beta d \gamma, \\
G_{z}= & -\int_{\mathbf{R}^{3}} \frac{\alpha}{\gamma} g_{x}(\alpha, \beta, \gamma, t) e^{2 \pi i(\alpha x+\beta y+\gamma z)} d \alpha d \beta d \gamma \\
& -\int_{\mathbf{R}^{3}} \frac{\beta}{\gamma} g_{y}(\alpha, \beta, \gamma, t) e^{2 \pi i(\alpha x+\beta y+\gamma z)} d \alpha d \beta d \gamma, \\
F_{1}= & \int_{\mathbf{R}^{2}} f_{x z}(\alpha, y, \gamma, t) e^{2 \pi i(\alpha x+\gamma z)} d \alpha d \gamma,
\end{aligned}
$$




$$
\begin{aligned}
& H_{1}=\int_{\mathbf{R}^{2}} f_{x z}(\alpha, y, \gamma, t) \frac{\alpha e^{2 \pi i(\alpha x+\gamma z)}}{\gamma} d \alpha d \gamma, \\
& F_{2}=\int_{\mathbf{R}^{2}} f_{y x}(\alpha, \beta, z, t) e^{2 \pi i(\alpha x+\beta y)} d \alpha d \beta, \\
& H_{2}=\int_{\mathbf{R}^{2}} f_{y x}(\alpha, \beta, z, t) \frac{\beta e^{2 \pi i(\alpha x+\beta y)}}{\alpha} d \alpha d \beta, \\
& F_{3}=\int_{\mathbf{R}^{2}} f_{y z}(x, \beta, \gamma, t) e^{2 \pi i(\beta y+\gamma z)} d \beta d \gamma, \\
& H_{3}=\int_{\mathbf{R}^{2}} f_{y z}(x, \beta, \gamma, t) \frac{\beta e^{2 \pi i(\beta y+\gamma z)}}{\gamma} d \beta d \gamma .
\end{aligned}
$$

Here, functions $g_{x}, g_{y}, f_{x z}, f_{y x}$ and $f_{y z}$ are integrable and differentiable over $\mathbf{R}^{3} \times$ $(0, a)$. It is clear that these functions are connected with the functions on the right-hand side of Eq. (22) by Fourier transformation. In particular for $g_{x}, g_{y}$ the transformation is 3-dimensional and 2-dimensional for others.

The physical condition (2) leads to the special form of functions $g_{x}, g_{y}, f_{x z}, f_{y x}$ $f_{y z}$. It is possible to construct the ansatz for the solution of the Euler equation for ideal fluid in the same way as presented here and by application of the physical condition (2).

\section{Nonlinear Autonomous ODE}

Let us consider the well-known case of second order autonomous dynamical system (which is called as Lienard system):

$$
\ddot{x}+\phi(x) \dot{x}+\psi(x)=0,
$$

for $t \in[0, a] \subset \mathbf{R}$. The goal is to derive a general solution of Eq. (24) or find a way to solve the Cauchy problem. The dynamical system determined by Eq. (24) is autonomous, i.e. its left-hand side does not contain the time $t$ explicitly. That is why the order of the system could be decreased

$$
y^{\prime}+\phi(x)+\frac{\psi(x)}{y}=0,
$$

where $y=y(x)=\dot{x} \neq 0$.

One can consider first-order ODE of the general type

$$
y^{\prime}=F(x, y)
$$

instead of (25) and make some relevant propositions. Determine the FDE corresponding to (26):

$$
D \hat{y}=F(x, \hat{y})+O(\delta),
$$

where $\lim _{\delta=0} \hat{y}(x, \delta)=y(x)$. 
Let us denote $x_{0} \equiv x(0)$ and $x=x(t)$. By using the definition of $D$ operation in ordinary way $\left(D \hat{y}=\frac{1}{\delta}(\hat{y}(x+\delta)-\hat{y}(x))\right)$, we have

$$
\hat{y}\left(x_{0}+\delta\right)=\hat{y}\left(x_{0}\right)+\delta F\left(x_{0}, \hat{y}\left(x_{0}\right)\right)+O\left(\delta^{2}\right) .
$$

Theorem 4.1. Let Eq. (27) have a solution $\hat{y}$ such that $\hat{y}(x, \delta)$ has the same sign as $\hat{y}\left(x_{0}, \delta\right)$ for every $x \in(x(0), x(a))$. Let $\hat{w}(x, \delta) \equiv-\ln \hat{y}(x, \delta)$. If the limit

$$
\lim _{\delta=0} \hat{w}(x, \delta)=w(x)
$$

exists and the sequence

$$
\left\{S_{n}\right\} \equiv\left\{\sum_{i=1}^{n} C_{k}^{n}\left(\frac{x-x_{0}}{n}\right)^{i} w^{(i)}\left(x_{0}\right)\right\},
$$

where $C_{k}^{n}$ are binomial coefficients, is convergent for every appointed variable $x$, then the new dependent variable

$$
w(x)=-\ln y(x)
$$

integrates the Eq. (26), but may be as a convergent range. Note that

$$
w^{\prime}=-\frac{F}{y}, \quad w^{(2)}=\frac{F^{2}-F^{\prime} y}{y^{2}}, \quad, w^{(3)}=\frac{-2 F^{3}+3 F^{\prime} F y-F^{\prime \prime} y^{2}}{y^{3}}, \ldots
$$

Proof. By splitting the segment $[x(0), x(t)], t \in(0, a]$, into $n$ equal parts, let each of them have length $\delta$. When $\delta$ go to zero, $n$ go to $\infty$. Using (28) $n$ times, we have

$$
\begin{aligned}
\hat{y}(x)= & \hat{y}\left(x_{0}+n \delta\right)=\hat{y}\left(x_{0}\right) \\
& +\delta \sum_{j=0}^{n-1} F\left(x_{0}+j \delta, \hat{y}\left(x_{0}\right)\right. \\
& \left.+\delta \sum_{k=0}^{j-1} \delta F\left(x+k \delta, y\left(x_{0}\right)+\delta F(x+k \delta)+O\left(\delta^{2}\right)\right)\right)+O\left(\delta^{2}\right) .
\end{aligned}
$$

It is evident that

$$
\hat{y}\left(x_{0}+\delta\right)=\delta D y\left(x_{0}\right)+y\left(x_{0}\right)=y\left(x_{0}\right)+\delta F\left(x_{0}, y\left(x_{0}\right)\right)+O\left(\delta^{2}\right)
$$

At the same time

$$
\begin{aligned}
& F\left(x_{0}, y\left(x_{0}\right)\right)=D \hat{y}\left(x_{0}\right)-O(\delta)=\frac{e^{-\hat{w}\left(x_{0}\right)}}{\delta}\left(e^{-\delta D \hat{w}\left(x_{0}\right)}-1\right), \\
& F\left(x_{0}+\delta, y\left(x_{0}\right)+\delta F\left(x_{0}\right)+O(\delta)\right)=\frac{e^{-\hat{w}\left(x_{0}+\delta\right)}}{\delta}\left(e^{-\delta D \hat{w}\left(x_{0}+\delta\right)}-1\right),
\end{aligned}
$$


and so on. Using identities

$$
\hat{w}(x+\delta)=\delta D \hat{w}(x)+\hat{w}(x), \quad D \hat{w}(x+\delta)=\delta D^{2} \hat{w}(x)+D \hat{w}(x),
$$

we derive

$$
\begin{aligned}
\hat{y}(x, \delta)= & \hat{y}\left(x_{0}+n \delta\right) \\
= & \hat{y}\left(x_{0}\right)\left[1+e^{-\delta D \hat{w}}-1+e^{-2 \delta D \hat{w}-\delta^{2} D^{2} \hat{w}}\right. \\
& \left.-e^{-\delta D \hat{w}}+\cdots+O\left(\delta^{2}\right)\right]
\end{aligned}
$$

where $\hat{w}=\hat{w}\left(x_{0}\right)$. The sum in Eq. (33) has a result

$$
e^{-\sum_{i=1}^{n+1} C_{i}^{n+1} \delta^{i} D^{i} \hat{w}} \text {. }
$$

That is why, (33) transforms to

$$
\ln \frac{\hat{y}(x, \delta)}{\hat{y}\left(x_{0}\right)}=-\sum_{i=1}^{n+1} C_{i}^{n+1} \delta^{i} D^{i} \hat{w} .
$$

Eq. (34) when $\delta \rightarrow 0$ comes to

$$
\ln \frac{y(x)}{\hat{y}\left(x_{0}\right)}=-\sum_{i=1}^{n+1} C_{i}^{n+1}\left(\frac{x-x_{0}}{n}\right)^{i} w^{(i)},
$$

because $\delta=\frac{x-x_{0}}{n}$, and it is assumed that $n \rightarrow \infty$. Equation (35) represents the limit of the sequence $S_{n+1}$ in (30) and hence it is convergent. That is, we have the relation (35) which is connecting $y(x)$ and $y\left(x_{0}\right)$ through $w^{(i)}\left(x_{0}\right)$, depending on $F^{(i)}\left(x_{0}\right)$ and $y\left(x_{0}\right)$ only. Consequently, we can say that Eq. (35) represents a Cauchy problem solution in the form of convergent range.

Let us use the result of this theorem to find the general solution of the Eq. (24). Note that it is evident that any solution of Eq. (26) could be presented as a range in some neighborhood of the point $x_{0}$, but the above Theorem shows that the variable change $w=-\ln y$ gets to the solution of the Cauchy problem not over the neighborhood but for any interval $(x(0), x(a)$ ) (up to sign of the function $y(x))$. That is why it is possible to use the new variable $w$ in order to solve the Eq. (25) over any interval which is in correspondence with the Theorem 4.1 conditions. Making the change of variables we obtain

$$
w^{\prime}=\phi e^{w}+\psi e^{2 w}
$$

For Lienard Equation (25) we have

$$
F(x, y)=-\phi(x)-\frac{\psi(x)}{y}
$$

and Eq.(27) takes the form

$$
D \hat{y}=-\phi(x)-\frac{\psi(x)}{\hat{y}}+O(\delta) .
$$


Using the theorem 4.1, we can derive that solution $y(x)$ of Eq. (25) has the following form

$$
y(x)=y\left(x_{0}\right) \exp \left[-\lim _{n=\infty} \sum_{i=1}^{n+1} C_{i}^{n+1}\left(\frac{x-x_{0}}{n}\right)^{i} w^{(i)}\right],
$$

where $w^{\prime}=\phi\left(x_{0}\right) / y\left(x_{0}\right)+\psi\left(x_{0}\right) / y^{2}\left(x_{0}\right)$ and

$$
w^{(i)}=\frac{d w^{(i-1)}}{d x}\left(x_{0}\right), \quad F_{x}^{\prime}\left(x_{0}\right)=-\phi^{\prime}\left(x_{0}\right)-\frac{\psi^{\prime}\left(x_{0}\right)}{y\left(x_{0}\right)}, \quad y^{\prime}\left(x_{0}\right)=F\left(x_{0}, y\left(x_{0}\right)\right) .
$$

Note that if the limit exists for given functions $\phi, \psi$ and the initial condition is $y_{0}=y\left(x_{0}\right)$, then Eq. (37) yields an exact solution of the Cauchy problem for Lienard dynamical system. This is convenient for computer modeling of the dynamical system. Of course, the case of Lienard system is a simple example. The result of the theorem 4.1 could be applied to any autonomous dynamical system of second order or to any first order dynamical system.

\section{Acknowledgments}

I appreciate Prof. Asya Aminova of the Kazan State University, Russia, for supervising my work several years ago. I also thank Prof. Asghar Qadir and Dr. Sajid Ali for inviting me to SDEA-II, paying attention to my work and initiating useful discussions.

\section{References}

1. J.R. Cannon, Encyclopedia of Mathematics and Its Applications, 23 1st ed., (1984).

2. J. Carlson, A. Jaffe, A. Wiles (eds.), The Millennium Prize Problems, Clay Mathematics Institute (American Mathematical Soc., 2006).

3. B. Van der Pol, Phil. Mag., ser. 6, 43, (1922)

4. B. Van der Pol, Phil. Mag., ser. 7, 2, (1926)

5. A.O. Gelfond, Calculus of Finite Differences, (Hindustan Pub. Corp., Delhi, 1971)

6. K.Yu. Dorokhova, S.V. Zuev II Proceedings of young scientists and specialists of the Belgorod region, 2, (Belgorod: Pub. Comp. Constanta, 2013) (in Russian) 